\title{
Knowledge, attitudes and practices among Lebanese Obstetricians and gynecologists toward Coronavirus Disease-2019 (COVID-19) and pregnancy
}

Dalal Youssef ( $\nabla$ dalalyoussef.esu@gmail.com )

Ministry of Public Health

Linda Abou Abass

Ministry of Public Health

Atika Berry

Ministry of Public Health

Janet Youssef

Zahraa University Medical Center

\section{Article}

Keywords: Knowledge, attitudes, practices, pregnancy, COVID-19, Obstetricians, gynecologists

Posted Date: May 26th, 2021

DOI: https://doi.org/10.21203/rs.3.rs-193232/v1

License: (c) (i) This work is licensed under a Creative Commons Attribution 4.0 International License.

Read Full License 


\section{Abstract}

Introduction: The pandemic of Coronavirus Disease 2019 (COVID-19) has seriously disturbed the daily life of the general population particularly the life of the pregnant women. Since the obstetricians and gynecologists (OBGYN) are often the primary health care providers during pregnancy, hence the importance of their critical role in preventing and managing COVID-19 infection in their patients.

Objectives: This study aims to assess the knowledge, attitudes and practices of OBGYN in order to identify existing gaps and to improve patient and occupational safety.

Methods: A cross-sectional study, using an online survey, was conducted during the rapid rise of COVID19 pandemic in Lebanon over the period extending between 20th October and 20th November 2020 among Lebanese OBGYNs. The analysis was performed using Statistical Package for Social Sciences (SPSS) software. Data collected is subjected to descriptive analysis. A good level of knowledge was defined when the percentage of correct answers reaches more than $80 \%$ of the respondents.

Results: A total of 279 OBGYNs participated in the survey of which $57 \%$ were males. The majority of them (64.2\%) were aged more than 45 years, married (79.9\%) with large work experience (70.3\%). Only $28.3 \%$ were reluctant to take care of COVID-19 patient. Most of them feared of contracting COVID-19 or transmitting COVID-19 to their family member due to occupational exposure and $42.3 \%$ felt overwhelmed. $62.7 \%$ of them considered that policies implemented by the ministry of public health are sufficient. The majority of OBGYNs had a good level of knowledge in different basic and specific domains related to COVID-19 and pregnancy. Furthermore, a good practice score in all relevant aspects (personal, clinic and patient) was revealed.

Conclusion: The high knowledge and practice scores, among Lebanese OBGYNs disclose a strong commitment from the part of these physicians to fulfill their responsibilities during this pandemic towards themselves and their patients.

\section{Introduction}

Novel coronavirus disease 2019 (COVID-19), which first emerged in China in December 2019, has turned into a worldwide disaster affecting all the countries (1).

This pandemic has seriously disturbed the daily life of the general population particularly the life of pregnant women $(2,3)$. The devastating and massive global spread of COVID-19 has lifted concerns about its impact on antenatal, neonatal and postnatal health care (4-6). Given that the pregnancy represents a period of additional uncertainty, the anxiety triggered by the COVID-19 itself, the accompanied forced social distancing, the financial difficulties and the sizeable changes in the provision of antenatal care are more pronounced in this particular group. Although current evidence revealed that pregnant women do not appear at higher risk to contract the infection than the general population (7). 
Owing to the sprouting of COVID-19 and the swiftly evolving nature of the pandemic, there is a dearth of high-quality evidence regarding pregnancy and COVID-19. Based on the available data, most COVID-19 pregnant women experienced mild or moderate cold/flu-like symptoms (8). However, intensive care admission may be more common in pregnant women with COVID-19 than in non-pregnant women of the same age (9). Furthermore, the risk of preterm birth is considered three times higher in pregnant woman with COVID-19 as well as increased rate of cesarean birth (10).

Concerning vertical transmission, current evidence discloses that the occurrence of a transmission antenatally or intrapartum from a woman to her newborn is uncommon. In case it arises, it seems not to be affected by mode of birth, method of feeding, or whether the woman and baby stay together (roomingin) (11). It is noteworthy that there has been no significant reported increase in the incidence of congenital abnormalities and there was no evidence of an increase in stillbirth or neonatal death among women with COVID-19, although the available evidence to comment on the risk of miscarriage is still scarce (11-14). The overall outcome of babies born to women with COVID-19 is positive and rarely acquired COVID-19 or show adverse clinical outcomes (15).

Given the potential risk of COVID-19 on pregnancy and the essentiality of antenatal and postnatal care, while witnessing social distancing and infection prevention measures, efforts were made at the international level to ensure the safety of pregnant women. This includes building the capacities of OBGYNs to respond proactively to the challenging healthcare needs during the pandemic. In this regard, guidance documents, training, and reports on pregnancy care during COVID-19, management of COVID19 pregnant women, maternity units with changes to services were developed and can be found on international websites of RCOG, ACOG, FIGO in order to assist and to support healthcare providers caring for these women $(11,16,17)$.

In Lebanon, the first documented case of COVID-19 infection in Lebanon was on February 21st, 2020 (18). Given the political and economic crisis that affects deeply the country, which hitherto dared with limited health system resources, the Lebanese population, were about to encounter another opponent revealed by the clinical course of COVID-19. As well, the particularity of the Lebanese demography linked to the presence of Syrian and Palestinian refugees, levy additional burden on health care system (19). Based on the Lebanese ministry of public health (MOPH) estimates, there is around 125 thousand Lebanese and non-Lebanese pregnant women with almost 50 thousand of them living with socioeconomic deprivation and/ or in crowded conditions (urban and camps settlements) (20). In this respect, national efforts were proactive to fight the spread of COVID-19 in the community. However, the COVID-19 pandemic spread swiftly with an upsurge of COVID-19 infection in the Lebanese community, shedding light on the care of pregnant women in Lebanon that require a unique and suitable response. With time, most of our public hospitals became majority of "COVID-19 hospitals". The demand for hospital beds and space increased rapidly, with considerable impacts on the functionality of the obstetrical service. In this respect, The MOPH found, since the early phase of the pandemic, a National Technical Committee on Corona and Pregnancy pointed to share technical expertise with health 
providers, notably OBGYN. Besides, it aims to raise awareness among the public regarding COVID-19 and pregnancy (21).

Since the OBGYN are often the only health care providers during pregnancy, hence the importance of their critical role in preventing COVID-19 in their patients through guidance, monitoring, raising awareness, and implementing recommended infection control practices as well as their role as counselors. However, this key role cannot be achieved effectively without capacitating and skilling OBGYN on the latest updates on COVID-19 and prevention measures in order to quickly address changing guidelines and evolving information.

To our knowledge, there are no studies in Lebanon assessing knowledge, attitudes and practices (KAP) of OBGYN toward COVID-19 and pregnancy. Thus, it is of great interest to conduct this study that aims to assess the knowledge, attitudes, and practices of OBGYN in order to identify existing gaps and to improve patient and occupational safety.

\section{Methods}

\section{Study design:}

A cross-sectional study, using an online survey, was conducted during the rapid rise of the COVID-19 pandemic in Lebanon over the period extending between 20th October and 20th November 2020. As the Lebanese government recommended the public to minimize face-to-face interaction, and this period includes the lockdown, potential respondents were electronically invited to participate.

\section{Instrumentation}

\section{Questionnaire development}

An extensive review of the literature was conducted to list available resources on KAP towards COVID-19 and antenatal care, as well as to identify relevant practices recommended for OBGYN during COVID-19. Most recent available information and guidelines on antenatal, neonatal, and postnatal care were reviewed $(7,11,19)$. A structured questionnaire was initially developed and designed by the authors to cover important aspects of knowledge, attitudes, fears, and, practice towards COVID-19 and pregnancy among OBGYN (Supplement file 1). The first draft of the questionnaire was reviewed by a panel of 5 experts that included a medical doctor, 2 epidemiologists, an infectious diseases expert, and an OBGYN to validate its contents with the intended constructs and theories. The panel selects the best items for clarity of the questions, interpretability, and accuracy of the knowledge, attitude, and practice domains. This panel also helped in identifying and evaluating the content validity (relevance, coverage, and representativeness) of the items in assessing the knowledge and practices. The content validity index $(\mathrm{CVI})$ was computed for each item, 3 items had a CVI below 0.8. A consensus was reached after omitting three items that were rated irrelevant. 
The original draft of the questionnaire was developed in English and then translated and adapted to the Arabic language. Therefore, the questionnaire was translated based on standard translation guidelines. It underwent two forward translations by a sworn translator and an epidemiologist. Subsequently, the two sets of translated Arabic questionnaires were subjected to a backward translation into English by another language communication expert as well as an epidemiologist. Minor linguistic edits were made (22). Face validity was conducted on 20 OBGYN to evaluate the comprehensibility and clarity of the items. A revised and finalized version of the questionnaire was produced from the findings of the face validation for use throughout the rest of the research. The questionnaire is self-administered and consisted of openended questions and was well received by participants. It was divided into five domains: (1) Sociodemography of participants; (2) knowledge of COVID-19 and pregnancy (3) Fears; (4) attitudes towards COVID-19 and government;(5) practices implemented

(1) Socio-demographic information including age, gender, marital status, number of children, household members, place of work, working in the frontline and clinical experience. Participants were also asked whether they have attended training about pregnancy in the era of COVID-19 and if they have been reluctant to take care of COVID-19 pregnant women.

(2) Knowledge section: Five dimensions with a total of 21 items were designed to measure OBGYN knowledge about basic information about COVID-19 (4 items), COVID-19 potential risk in pregnancy (4 items), Antenatal care and COVID-19 (7 items), Delivery and COVID-19 (4 items), Breastfeeding and COVID-19 (2 items). All the items were answered on a true/false basis and an additional "do not know" option. A correct response had a value of ' 1 ' and a "wrong" or don't know response had a value of ' 0 '. Hence, the cumulative score for all 21 knowledge questions would range from 0 to 21 points. Participants 'overall knowledge was categorized using modified Bloom's cut-off point, as good if the score was between 80 and 100\% (17-21 points), moderate if the score was between 50 and 79\% (11-16 points), and poor if the score was less than $50 \%$ ( $<10$ points)

(3) Fears section: The fears section comprises 4 items. Responses to questions related to fear were graded on a 3-point Likert scale, a scale ranging from "1" not afraid, "2" neutral and " 3 " for afraid.

(5) Attitudes section: The attitudes section comprises 2 domains were assessed using 6 questions to measure OBGYN: 1) attitudes toward government and health facility 2) attitudes toward COVID-19 pregnant women. Responses to questions related to risk perception were graded on a 3-point Likert scale, an agreement scale ranging from ' 1 ' for disagree to ' 3 ' for agree.

(6) Practice section: Three main domains constitute this section and 21 questions were used to evaluate the good practices. The first one was about taking preventive measures when heard about COVID-19 (1 item). For this question, the item was answered "yes" and "no" The second one is related to the basic infection prevention and control measures at the personal level (5 items) and the second one is about OBGYN specific practices at the clinic level (14 items) and the third domain is about practices of OBGYNs with the pregnant woman ( 6 items). The items of these 3 domains were answered "always", "occasionally" and "never". 
The questionnaire was validated by doing a pre-test on $5 \%$ of the sample before the actual data collection period. Survey flow, functionality and language were based on the pre-test feedback. Furthermore, the reliability of the questionnaire was checked, and their Cronbach Alpha value was 0.82 . The average time for filling the survey was 9 minutes.

\section{Sample size calculation}

To calculate the sample size of the study, Raosoft sample size calculator designed specifically for population surveys was used. Based on the list of physicians, currently registered as OBGYN, provided by Lebanese order of physicians, there is approximately 2000 registered OBGYN, less than 1000 of them are currently active and practicing at health facilities level, a 95\% confidence level was used and an absolute error was estimated to be $5 \%$. The required sample size was 278 OBGYN. The closure of response acceptance was done upon reaching of the target sample size.

\section{Data collection}

An online questionnaire using a Google form was emailed to all registered OBGYN in both Lebanese syndicates of doctors in North and Beirut. Then, OBGYNs were contacted via phone call and notified about the survey and its purpose. Upon their agreement to participate, the link of the study was sent through "WhatsApp" or email. This link corresponding to the survey includes a brief introduction to the background, the objective of the survey, and instructions for filling the questionnaire.

Exclusion criteria included: retired physicians, those who were out of the country at the time of the survey, as well as those not practicing actually. OBGYN who were not reachable due to change of their contact information during the time of the survey and those who refused to participate in the study were also excluded.

\section{Ethical considerations}

Participants were informed that all information would be gathered anonymously and handled confidentially. Participation was voluntary, and the questionnaire was collected only in subjects who expressed consent for study participation. As individual participants cannot be identified based on the presented material, this study caused no plausible harm or stigma to participants. The study design assured adequate protection of study participants, and neither include clinical data about patients nor configure itself as a clinical trial.

\section{Statistical analysis}

The collected data were checked for completeness and consistency before analysis. The data were analyzed using the statistical software SPSS (Statistical Package for Social Sciences), version 22.0. A total of 5 Scores was computed: Knowledge, perception, fears, attitudes, and practices. A reliability analysis was realized to validate each of these scores was performed using the Cronbach's alpha test (Cronbach Alpha should be more than 0.7). Descriptive statistics were reported using frequency and 
percentages for categorical variables. Finally, the analyzed data were organized and presented in the tabular, graphical and narrative form as necessary.

\section{Results}

\section{1- Baseline characteristics of the study participants}

Table 1 shows the baseline characteristics of the participants. A total of 279 OBGYNs participated in the survey of which $57 \%$ were males. The majority of them (64.2\%) was aged more than 45 years and was married (79.9\%). Regarding their household, the majority of them (78.5\%) have children at home, $26.2 \%$ of them were living with a family members having underlying conditions and $34.4 \%$ of them were living with an elderly aged $>65$ years.

Of the total, $38 \%$ of participating OBGYNs are working in the frontline during COVID-19 pandemic. With respect to their work, the majority of them (70.3\%) had a large work experience (more than 10 years) and are working in private hospitals (78.1\%) and clinics (86.7\%). Only $21.5 \%$ of them had a previous experience during an epidemic. Moreover, $39.8 \%$ of them have completed previous training about COVID19. It is noteworthy, that $51.3 \%$ of them have treated COVID-19 cases and only $28.3 \%$ were reluctant to take care of COVID-19 pregnant women.

\section{Knowledge:}

\section{1-OBGYNs self-reported knowledge}

The majority of OBGYNs (65.6\%) had an overall good level of knowledge $(\geq 80 \%)$ and only $1 \%$ of them had a poor level of knowledge $(<60 \%)$. Table 2 illustrates the knowledge domains about COVID-19 and pregnancy among OBGYNs. The majority of respondents were knowledgeable in different domains. The highest knowledge scales were shown in the general knowledge about COVID-19 and the domain related to the delivery where OBGYNs had a knowledge level $91.8 \%$ of and $95 \%$ respectively. Besides, $77.1 \%$ of respondents were well informed about the specific knowledge concerning pregnancy and COVID-19 akin to the medicinal treatment of COVID-19 knowledge domain (72.4\%). Correspondingly, the majority of them (78.9\%) had good knowledge concerning breastfeeding and COVID-19.

Table 3 describes OBGYNs answers towards pregnancy and COVID-19 knowledge items. Despite the good level of knowledge recorded in the domain related to the specific knowledge regarding pregnancy and COVID-19, only $41.2 \%$ of OBGYNs were aware that the overall risk of COVID-19 infection in the pregnant patient is low. Also, around $40 \%$ of OBGYNs were not knowledgeable that pregnant patients with COVID-19 could develop respiratory complications requiring intensive care more than COVID-19 positive non-pregnant patients.

In respect of the medicinal treatment of COVID-19, only $59.9 \%$ of participants recognized that high doses of systemic corticosteroids should be avoided in confirmed or suspected COVID-19 cases and $29.4 \%$ were not well informed that the use of non-steroidal anti-inflammatory drugs (NSAID), like ibuprofen, could 
worsen COVID-19 status. It is noteworthy that $26.9 \%$ of respondents consider cesarean section as an indication for delivery of all pregnant COVID-19 patients and 17.6\% were not cognizant that COVID-19 cannot be transmitted through breast milk.

\section{Fears of OBGYN's respondents toward COVID-19}

Table 4 describes OBGYNs answers towards items related to fear of COVID-19. Among the 279 practicing OBGYNs, almost $74.6 \%$ declared being afraid of contracting COVID-19 due occupational exposure and almost all of them (95\%) were afraid of transmitting COVID-19 to their family member in case they got infected. Almost $64.2 \%$ of respondents were afraid of being very sick in case they catch COVID-19. Out of 279 OBGYNs, less than half of OBGYNs unveiled that they were afraid of working in facilities where COVID-19 positive patients are admitted and followed.

\section{Impact of COVID-19 on OBGYNs well-being}

Table 5 summarizes OBGYNs answers regarding the impact of COVID-19 on their well-being. Only 27.2\% declared that their anxiety and worries are affecting the quality of care anticipated to the patient. However, nearly half of them (42.3\%) felt overwhelmed and burned out by the workload during COVID-19 pandemic.

\section{Attitudes of OBGYNs toward pregnant woman with COVID-19}

The majority of OBGYNs (76\%) considered that some pregnant patients will hide their exposure to COVID19 from them and $81 \%$ of them will hide their positivity from their social environment in order to avoid stigmatization. Nearly $84 \%$ of the participants highlighted the importance of supporting the mental status of the pregnant patient with COVID-19 during antenatal care (Figure 1).

\section{Attitudes of OBGYNs towards preventive measures adopted by government and health facilities}

The majority of respondents (72\%) believed that measures limiting the traffic of OBGYNs and medical staff between health facilities are rational and $62.7 \%$ of them declared that policies and actions implemented by the ministry of public health are sufficient and adequate whereas only $51.9 \%$ thought that the preventive measures applied by their health care facilities were appropriate and sufficient in fighting COVID-19 and to protect health care workers (Figure. 2).

\section{Practices:}

\section{Practices domains:}

All the OBGYNs declared that they made change to their practices since the emergence of COVID-19. The majority of them (80.3\%) had a high and good overall practice score. The highest score was observed in the domain related to the practices at personal level (93.5\%) followed by the practices concerning counseling and raising awareness about COVID-19 among pregnant women (86.7\%). At clinic level, 78.9\% had good practice score (Figure 3) 
Table 6 displayed the items of the practice score. Overall, the OBGYNs have good practices at different levels. $58.1 \%$ of them limit duration of the consultation to the just necessary all the time while $31.5 \%$ did it occasionally. During COVID-19, 35.5\% of respondents switched completely to the use of telehealth modalities and $60.6 \%$ performed it occasionally. Almost the majority of OBGYNs preserved and prioritized in-person visits for patients with greatest medical needs for in-person examination and they postpone non-emergent gynecologic/well women appointments. In the regard of awareness of counseling, the majority of OBGYNs counsel pregnant woman about the potential risk of COVID-19, document their discussion regarding prenatal care plan in her medical record and emphasize measures to prevent COVID19 for pregnant woman and their families. They also encourage woman to maintain prenatal care appointments and to took needed vaccines during pregnancy and to notify him and health authorities about potential exposure to COVID-19. Few of them distribute brochures and guidelines regarding COVID19 and pregnancy.

\section{Discussion}

This study was conducted during the rise of the COVID-19 outbreak in Lebanon which is accompanied by a concomitant increase of pregnant women infected by COVID-19. To the best of our knowledge, this is the first national Lebanese study aiming to explore OBGYNs knowledge, attitudes and practices toward COVID-19 and, antenatal care. It also reveals OBGYN's insights, doubts and, fears, hence allowing us to better understand the gaps and weak aspects in practices to be target in future interventions. A Similar topic was addressed by Awad et al., from the pregnant women point of view (23).

\section{Baseline Characteristics}

The baseline characteristics of the participants in our study defined a representative sample of Lebanese OBGYNs. Its main findings that the majority of respondents had a large experience (>10 years) and $38 \%$ of participating OBGYNs are working in the frontline during COVID-19 pandemic). Only $21.5 \%$ of them had a previous experience during an epidemic. Around half of OBGYNs have treated COVID-19 cases.

A finding of significant concern in this survey is that more than $28.3 \%$ of the respondents expressed their reluctance towards treating a pregnant woman with COVID-19. Given the unpredictability, novelty and infectiousness of COVID-19, in addition to the scarcity of data related to COVID-19 and pregnancy, the reluctance of OBGYNs should not be surprising and could be understood. However, this caused a moral dilemma between a duty to treat and a right to refrain. Our findings are consistent with a study conducted in Nepal where $35.9 \%$ of HCWs showed an unwillingness to work during the pandemic (24). In Bangladesh, it is reported that the reluctance of some physicians to treat patients suspected of COVID-19 infection, was due to personal risk assessment and not to reliable objection (25). Indeed, such hesitancy is not surprising as frontline health care workers have "more than three times the risk of COVID-19 infection than the general public" (26). However, these results highlight the importance of exploring the determinants of reluctance among Lebanese OBGYNs. 
More than half of OBGYNs' respondents have cared for a COVID-19 woman which is a percentage to take considerably, given that COVID-19 cases escalate in Lebanon during the study period where wider community transmission is observed.

\section{Knowledge:}

The majority of OBGYNs (65.6\%) had an overall good level of knowledge and only $1 \%$ of them had a poor level of knowledge (<60\%). This result is in line with that of Abou Abass et al., who reported that $89.5 \%$ of Lebanese physicians have a good knowledge regarding COVID-19 (27). Future plans should focus on improving the knowledge of OBGYNs with moderate knowledge levels. When looking in-depth at the knowledge domains, we found that most OBGYNs were cognizant of the different domains.

Most of OBGYNs were well-informed and updated in different knowledge domains. The highest knowledge scales were shown in the general knowledge part and the domain related to the delivery where OBGYNs had a knowledge level $91.8 \%$ of and 95\% respectively. Despite the good level of knowledge specific to pregnancy and COVID-19, only $41.2 \%$ of OBGYNs were aware that the overall risk of COVID-19 infection in the pregnant patients is low. However, a new study from the University of Texas Southwestern has found that pregnant women who test positive for COVID-19 and their newborn babies have a low risk of developing severe symptoms (28).

In addition, around 40\% of OBGYNs were not knowledgeable that pregnant patients with COVID-19 could develop respiratory complications requiring intensive care more than COVID-19 positive non-pregnant patients. This could be due to the swift generation of knowledge and its continuous update based on the availability and the emergence of new evidence based on the evolving nature of the disease which requires constant and regular upgrading of OBGYNs' knowledge.

The breach in knowledge was ostensible in the domain related to the medicinal treatment of COVID-19, where only, nearly half of surveyed OBGYNs recognized that high doses of systemic corticosteroids should be avoided in confirmed or suspected COVID-19 cases. The divulged poor knowledge in the medicinal treatment domain was particularly related to the question concerning the use of non-steroidal anti-inflammatory drugs (NSAID), as ibuprofen, which could worsen COVID-19. However, to date, most COVID-19 clinical trials have excluded or included very few pregnant and lactating women. This limitation makes it difficult to generate evidence-based recommendation on the treatment of COVID-19 and potentially limit the treatment options $(29,30)$. Peculiar finding in this study that $26.9 \%$ of respondents consider cesarean section as an indication for delivery of pregnant COVID-19 patients.

In addition, $17.6 \%$ were not conscious that COVID-19 cannot be transmitted through breast milk. This could be attributed to the scientific dilemma anticipated by the experts regarding this topic and the evolving nature of the disease.

\section{Fears:}


The present study also revealed that more than $74.6 \%$ of the surveyed OBGYNs were afraid of getting infected with COVID-19 due to their occupational exposure, and approximately all of them were afraid of transmitting COVID-19 to their family member in the case they got infected. Our results are in line with the findings of studies conducted in Lebanon, Egypt and, Denmark $(27,31,32)$.

More than half of respondents feared being very sick in case they catch COVID-19 and many of them were frightened of working in facilities where COVID-19 positive patients are admitted and followed. This could be attributed to the fact that $37 \%$ of participants were aged more than 55 years old and the complications of COVID-19 anticipated with age (33). Indeed, a high range of anxiety and burnout were expected since this outbreak impacted every and daily personal life across this globe especially the aspects of life of health care providers. The current financial crisis in Lebanon heightens the situation. Thus, addressing OBGYN's fears, and perceptions through psychological intervention would be necessary to help them to cope with daily stressors during the outbreak (34). Such finding stressed the importance of building and consolidating OBGYNs' confidence which is of paramount importance not only for the physicians at a personal level but for effective practice revealed by the increase of their willingness to treat COVID-19 pregnant women.

\section{Impact of COVID-19 on OBGYN wellbeing:}

COVID-19 has sternly negatively impacted the wellbeing of OBGYNs. Our findings revealed that $27.2 \%$ of OBGYNs considered that their anxiety and worries are affecting the quality of care anticipated to the patient and $42.3 \%$ of them felt overwhelmed and burned out by the workload during COVID-19 pandemic. Our findings were in line with the results displayed in many studies. A survey conducted in India about burnout among HCW during COVID-19 pandemic shown that the prevalence of personal burnout was $44.6 \%$ (903), and $52.8 \%$ had pandemic-related burnout (35). These results also shed light on the importance of perusing in-depth mental health issues among OBGYNs.

\section{Attitudes:}

Concerning attitudes of OBGYNs towards government approach and response to COVID-19 pandemic, interestingly, $62.7 \%$ of the participants declared that policies and actions implemented by the ministry of public health. Our findings showed a decrease in the positive attitude towards the government response in comparison with a previous study conducted at the early phase of the pandemic among Lebanese physicians (27). However, the success of measures (lockdown, point of entry closure, school closure, curfew...) showed in the early phase in containing the outbreak, was time-bounded. It vanished gradually with the release of lockdown and the situation was aggravated after Beirut blast where a spike of cases was reported.

Nevertheless, only $51.9 \%$ of OBGYNs thought that the preventive measures applied by their health care facilities were appropriate and sufficient in fighting COVID-19 and to protect health care workers. This highlights the importance of improving infection prevention practices within the health facilities. On another hand, the majority of respondents ( $72 \%$ ) believed that measures limiting the traffic of OBGYNs 
and medical staff between health facilities are rational since restraining the work circle could control the spread of COVID-19.

\section{Attitudes of OBGYNs toward the pregnant woman with COVID-19}

Surprisingly, a large number of surveyed OBGYNs admitted that some pregnant patients will hide their exposure to COVID-19 from them. Even though, the majority $81 \%$ of OBGYNs perceived that pregnant woman will hide their positivity from their social environment in order to avoid stigmatization (36). However, social stigma of contracting COVID-19 motivates the general public not to disclose possible infection when presenting for medical care and, therefore, place physicians and nurses at risk (37). Hence, $84 \%$ of the participants highlighted the importance of supporting the mental status of the pregnant patient with COVID-19 during antenatal care which is consistent with Awad et al results (23).

\section{Practices:}

Interestingly, all surveyed OBGYNs acknowledged changes since the early phase of the pandemic. The majority of them (80.3\%) had a good overall practice score. The highest score was observed in the domain related to the practices at a personal level (93.5\%) which included regular hand hygiene, wearing PPEs including masks and gloves, changing gloves after each patient and, regular cleaning and disinfection of surfaces and equipment's (especially high touched surfaces). A study conducted in Bangladesh revealed that the proper use of face shields or goggles in addition to decontamination could significantly protect the physicians from COVID-19 (38). Such proper implementation of good practices would mitigate this problem(39).

Based on OBGYNs responses, $78,9 \%$ of them had a good practice score at the clinic level. These good practices including requesting all the time from all visitors to wear a mask $(98.2 \%)$ and requiring that all health staff at the clinic wear protective clothing (75.6\%). These practices would enhance patient confidence and alleviate their fears during the pandemic.

Limiting the duration of the consultation strategy was adopted by OBGYNs. The majority of OBGYNs followed a reducing in-person visit and employing an extended hours strategy and $90.1 \%$ of them have made some modifications to the waiting room and clinic space to accommodate physical distancing. Also, they prioritized in-person visits for patients with the greatest medical needs for in-person examination while postponing non-emergent gynecologic or well women appointments. Hence, compliance and adherence of the OBGYNs to ACOG and other OBGYN societies recommended measures was well noticed.

The bulk of OBGYN performed pre-appointment screening of patients for in-person visits concerning potential exposure to COVID-19, symptoms and, temperature checking. Such measures are essentials to ensure patient and physician safety.

Since providing telehealth services was essential to their mission as physicians during the pandemic, shifting to Telemedicine and maximizing the use of the telehealth modalities (calls, video calls...) was 
acknowledged by a large number of OBGYNs. Consistently to the findings of a study conducted by Helou et al, regarding the use of telehealth by physicians in Lebanon showed an increase in physicians use of telehealth activities during the COVID-19 pandemic in the fields of telemedicine (40). Reliable remote payment and reimbursement services may prove to be an important barrier shortly, due to the uncertainty of Lebanon's economy and lack of concomitant legislation.

\section{Raising awareness and counseling:}

Moreover, $86,7 \%$ of OBGYNs had a good practice score concerning their role in raising awareness and counseling. Also, they emphasized measures to prevent COVID-19 and their families Adherent to ACOG and other OBGYN societies' recommendations, most OBGYNs instructed their patients to maintain prenatal care appointments and to take needed vaccines during pregnancy. This would reduce the misbeliefs of pregnant women during this critical period. However, poor adherence to the influenza vaccine has been noticed among Lebanese pregnant women (23). Despite that the majority of OBGYNs advised their patients, it is astonishing that $16.8 \%$ of them didn't enlighten these methods. Our results were inconsistent with the study conducted among Lebanese pregnant women who reported that half of participants did not discuss methods of contraception with their physicians (23).

However, brochures, guidelines regarding COVID-19 and pregnancy were not distributed by nearly half of OBGYNs. This can be due to their availability at their clinics or health facilities due to inaccessibility and financial issue.

Considering the fear of the social stigma that may lead some pregnant women to hide their exposure and positivity for COVID-19, the majority of OBGYNs my patients to notify me and the health authorities of any potential exposure to COVID-19 (41).

\subsection{Study limitations}

Some limitations of this study should be acknowledged. First, our study relies on OBGYNs' self-reported information, which may be a threat to internal validity. In fact, self-reporting could be influenced by social desirability and then intentionally modified to meet the norms. Furthermore, this online questionnaire might have favored a selection bias by since it might only allow the participation of OBGYN users who have access to online resources to participate. The findings of the present study should be considered in light of several limitations. No validated tool for the assessment of the knowledge, attitudes and, practices of OBGYNs was available. We have formulated items from ACOG guidelines. Based on a study exploring the characteristics of healthcare providers in Lebanon, less than $20 \%$ of Lebanese physicians are female (42), since then, an increase in the percentage of female physicians was noticed (current estimate of 25\%). The demographic characteristics of our respondents show a higher percentage of male physicians ( $57 \%$ male vs. $43 \%$ female). The slight overrepresentation of female physicians in our sample could be attributed to our sampling method and the ability of participation of females more than males in such surveys. 


\section{Conclusion}

In conclusion, a high knowledge and practice scores, among Lebanese OBGYNs reveal a strong commitment from the part of these physicians to fulfill their responsibilities towards themselves, their patient, and their society to confront this pandemic. Knowledge of aspects specific to COVID-19 and gestation by OBGYNs allow them to correctly diagnose the disease, and take the most appropriate management decisions. Implementing and adopting preventive measures are essential for providing appropriate antenatal, intrapartum, and postnatal care particularly during this wider community transmission. Keeping up-to-date and understanding every limitation during the pregnancy will prevent further complications and alleviate the burden on the health care system especially during this explosion phase of COVID-19.

\section{References}

1. Zhu H, Wei L, Niu P. The novel coronavirus outbreak in Wuhan, China. Glob Health Res Policy. 2020;5:6-.

2. Kajdy A, Feduniw S, Ajdacka U, Modzelewski J, Baranowska B, Sys D, et al. Risk factors for anxiety and depression among pregnant women during the COVID-19 pandemic: A web-based crosssectional survey. Medicine. 2020;99(30):e21279.

3. Haleem A, Javaid M, Vaishya R. Effects of COVID-19 pandemic in daily life. Curr Med Res Pract. 2020;10(2):78-9.

4. Coxon K, Turienzo CF, Kweekel L, Goodarzi B, Brigante L, Simon A, et al. The impact of the coronavirus (COVID-19) pandemic on maternity care in Europe. Midwifery. 2020;88:102779-.

5. Bick D, Cheyne H, Chang Y-S, Fisher J. Maternal postnatal health during the COVID-19 pandemic: Vigilance is needed. Midwifery. 2020;88:102781-.

6. Kimani RW, Maina R, Shumba C, Shaibu S. Maternal and newborn care during the COVID-19 pandemic in Kenya: re-contextualising the community midwifery model. Human Resources for Health. 2020;18(1):75.

7. The American College of Obstetricians and Gynecologists (ACOG). Latest update 14 December 2020. Available https://www.acog.org/clinical/clinical-guidance/practice-advisory/articles/2020/03/novelcoronavirus-2019 Accessed 20 December 2020.

8. López M, Gonce A, Meler E, Plaza A, Hernández S, Martinez-Portilla RJ, et al. Coronavirus Disease 2019 in Pregnancy: A Clinical Management Protocol and Considerations for Practice. Fetal Diagnosis and Therapy. 2020;47(7):519-28.

9. Ellington S, Strid P, Tong VT, et al. Characteristics of Women of Reproductive Age with LaboratoryConfirmed SARS-CoV-2 Infection by Pregnancy Status - United States, January 22-June 7, 2020. MMWR Morb Mortal Wkly Rep 2020;69:769-775. DOI:

http://dx.doi.org/10.15585/mmwr.mm6925a1external icon. 
10. Woodworth KR, Olsen EO, Neelam V, et al. Birth and Infant Outcomes Following Laboratory-Confirmed SARS-CoV-2 Infection in Pregnancy - SET-NET, 16 Jurisdictions, March 29-October 14, 2020. MMWR Morb Mortal Wkly Rep 2020;69:1635-1640. DOI: http://dx.doi.org/10.15585/mmwr.mm6944e2external icon.

11. Royal College of Obstetricians and Gynecologists. Coronavirus (COVID-19) Infection in Pregnancy. Accessed 7 Decemeber 2020. Available https://www.rcog.org.uk/globalassets/documents/guidelines/2020-10-14-coronavirus-COVID-19infection-in-pregnancy-v12.pdf.

12. Stowe J, Smith H, Thurland K, Ramsay ME, Andrews N, Ladhani SN. Stillbirths During the COVID-19 Pandemic in England, April-June 2020. JAMA. 2021;325(1):86-7.

13. Handley SC, Mullin AM, Elovitz MA, Gerson KD, Montoya-Williams D, Lorch SA, et al. Changes in Preterm Birth Phenotypes and Stillbirth at 2 Philadelphia Hospitals During the SARS-CoV-2 Pandemic, March-June 2020. JAMA. 2021;325(1):87-9.

14. Akhtar H, Patel C, Abuelgasim E, Harky A. COVID-19 (SARS-CoV-2) Infection in Pregnancy: A Systematic Review. Gynecologic and Obstetric Investigation. 2020;85(4):295-306.

15. Kyle MH, Glassman ME, Khan A, Fernández CR, Hanft E, Emeruwa UN, et al. A review of newborn outcomes during the COVID-19 pandemic. Semin Perinatol. 2020;44(7):151286-.

16. Coronavirus COVID-19, a practice advisory. [Available from https://www.acog.org/clinical/clinicalguidance/practice-advisory/articles/2020/03/novel-coronavirus-2019 access 20 December 2020.

17. International Federation Of gynecology and Obstetrics. Available https://www.figo.org/resources/COVID-19-resources. Accessed 7 December 2020.

18. MOPH. (2020) Epidemiological surveillance program of COVID-19. Retrieved from https://www.moph.gov.lb/en/Pages/2/24870/novel-coronavirus-2019.

19. International Labour Organization; ILO. Assessment of the impact of Syrian refugees in Lebanon and their employment profile 2014. Available https://www.ilo.org/wcmsp5/groups/public/--arabstates/-ro-beirut/documents/publication/wcms_240134.pdf. Accessed 7 December 2020.

20. Ministry of Public Health, MOPH, Lebanon. Available https://www.moph.gov.lb/en.

21. El Kak F. COVID-19 and pregnancy: Lebanon preparedness within global response. J Med Liban 2020; 68 (1-2) : 60-62.

22. Beaton DE, Bombardier C, Guillemin F, Ferraz MB. Guidelines for the Process of Cross-Cultural Adaptation of Self-Report Measures. Spine. 2000;25(24):3186-91.

23. Diana Carolina Awad, Aline Zaiter, Peter Ghiya, Karine Zaiter, Jean G Louka, Chadi Fakih, Mirna N Chahine. COVID-19: Pregnant Women's Knowledge, Perceptions \& Fears. First National Data from Lebanon. Obstetrics and Gynecology Research 3 (2020): 220-234.

24. Upadhyaya DP, Paudel R, Acharya D, Khoshnood K, Lee K, Park J-H, et al. Frontline Healthcare Workers' Knowledge and Perception of COVID-19, and Willingness to Work during the Pandemic in Nepal. Healthcare (Basel). 2020;8(4):554. 
25. Andalou Agency. Doctors plight mounting in Bangladesh amid pandemic https://www.aa.tr/en/asiapacific/doctors-plight-mounting-in-bangladeshamid-pandemic/1929449, Accessed 05 Aug. 2020.

26. Center for Infectious Disease Research and Policy, University of Minnesota.

27. Health workers, especially minorities, at high risk of COVID, even with PPE. https://www.cidrap.umn.edu/news-perspective/2020/08/health-workersespecially-minorities-highrisk-covid-even-ppe, Accessed 06 Aug. 2020.

27. Abou-Abbas L, Nasser Z, Fares Y, Chahrour M, El Haidari R, Atoui R. Knowledge and practice of physicians during COVID-19 pandemic: a cross-sectional study in Lebanon. BMC Public Health. 2020;20(1):1474.

28. Adhikari EH, Moreno W, Zofkie AC, MacDonald L, Mclntire DD, Collins RRJ, et al. Pregnancy Outcomes Among Women With and Without Severe Acute Respiratory Syndrome Coronavirus 2 Infection. JAMA Network Open. 2020;3(11):e2029256-e.

29. COVID-19 Treatment Guidelines Panel. Coronavirus Disease 2019 (COVID-19) Treatment Guidelines. National Institutes of Health. Available at https://www.covid19treatmentguidelines.nih.gov/. Accessed 7 December 2020.

30. Favilli A, Mattei Gentili M, Raspa F, Giardina I, Parazzini F, Vitagliano A, et al. Effectiveness and safety of available treatments for COVID-19 during pregnancy: a critical review. J Matern Fetal Neona. 2020:1-14.

31. Nabe-Nielsen K, Nilsson CJ, Juul-Madsen M, et alCOVID-19 risk management at the workplace, fear of infection and fear of transmission of infection among frontline employees. Occupational and Environmental Medicine Published Online First: 19 October 2020. doi: 10.1136/oemed-2020-106831.

32. Abdel Wahed WY, Hefzy EM, Ahmed MI, Hamed NS. Assessment of Knowledge, Attitudes, and Perception of Health Care Workers Regarding COVID-19, A Cross-Sectional Study from Egypt. J Community Health. 2020;45(6):1242-51.

33. Karlsson U, Fraenkel C-J. COVID-19: risks to healthcare workers and their families. BMJ. 2020;371:m3944.

34. Shanafelt T, Ripp J, Trockel M. Understanding and Addressing Sources of Anxiety Among Health Care Professionals During the COVID-19 Pandemic. JAMA. 2020;323(21):2133-4.

35. Khasne RW, Dhakulkar BS, Mahajan HC, Kulkarni AP. Burnout among Healthcare Workers during COVID-19 Pandemic in India: Results of a Questionnaire-based Survey. Indian J Crit Care Med. 2020;24(8):664-71.

36. Zaman S, Rahman S. Fear and stigma in the context of corona epidemic in Bangladesh. ProthomAlo. 2020; https://en.prothomalo.com/opinion/analysis/fear-and-stigma-in-the-context-of-coronaepidemic-in-bangladesh. Accessed 7 December 2020.

37. Swazo NK, Talukder MMH, Ahsan MK. A Duty to treat? A Right to refrain? Bangladeshi physicians in moral dilemma during COVID-19. Philosophy, Ethics, and Humanities in Medicine. 2020;15(1):7. 
38. Khalil MM, Alam MM, Arefin MK, Chowdhury MR, Huq MR, Chowdhury JA, et al. Role of Personal Protective Measures in Prevention of COVID-19 Spread Among Physicians in Bangladesh: a Multicenter Cross-Sectional Comparative Study. SN Compr Clin Med. 2020:1-7.

39. World Health Organization. Infection prevention and control during health care when novel coronavirus ( $\mathrm{nCoV}$ ) infection is suspected: interim guidance, 25 January 2020.

40. Helou S, Helou E, Abou Khalil V, Wakim J, Helou J, Daher A, et al. The Effect of the COVID-19 Pandemic on Physicians' Use and Perception of Telehealth: The Case of Lebanon. International Journal of Environmental Research and Public Health. 2020;17:4866.

41. American College of Obstetricians and Gynecologists. Novel Coronavirus 2019. Summary of Key Updates (December 14, 2020). Available https://www.acog.org/clinical/clinical-guidance/practiceadvisory/articles/2020/03/novel-coronavirus-2019. Accessed 20 December 2020.

42. Kassak KM, Ghomrawi HMK, Osseiran AMA, Kobeissi H. The providers of health services in Lebanon: a survey of physicians. Human Resources for Health. 2006;4(1):4.

\section{Declarations}

\section{Ethics approval and consent to participate:}

Research was performed in line with the principles of the Declaration of the Helsinki as revised in 2008. Written informed consent was obtained from all the participants at recruitment. Participants were informed that all information would be gathered anonymously and handled confidentially. Participation was voluntary, and the questionnaire was collected only in subjects who expressed consent for study participation. As individual participants cannot be identified based on the presented material, this study caused no plausible harm or stigma to participants. The study design assured adequate protection of study participants, and neither includes clinical data about patients nor configures itself as a clinical trial

\section{Funding}

No funding was received.

\section{Availability of data and materials}

The datasets used and analyzed during the current study will not be made publically available but will be available from the corresponding author on reasonable request.

\section{Consent for publication}

Not applicable. 


\section{Competing interests}

The author(s) declare that they have no competing interests.

\section{Authors' contributions}

DY developed the project idea. DY, JY and LAA formulated the questionnaire, organized and analyzed the survey. DY, JY, LAA, AB drafted and critically reviewed the paper. All authors read and agreed on the final version.

\section{Acknowledgements}

The authors would like to thank Mrs. Ola Issa for her help during the data collection. The authors are also grateful to all OBGYNs who accepted to be part of this study.

\section{Tables}


Table 1: baseline $(\mathrm{N}=279)$

n $\quad \%$

\section{Gender}

\begin{tabular}{|lll|}
\hline Male & 159 & $57.0 \%$ \\
\hline Female & 120 & $43.0 \%$ \\
\hline $25-34$ & & \\
\hline $35-44$ & 33 & $11.8 \%$ \\
\hline $45-54$ & 67 & $24.0 \%$ \\
\hline$\geq 55$ & 75 & $26.9 \%$ \\
\hline Marital status & 104 & $37.3 \%$ \\
\hline Single & & \\
\hline Married & 38 & $13.6 \%$ \\
\hline Other & 223 & $79.9 \%$ \\
\hline Presence of children & 18 & $6.5 \%$ \\
\hline Yes & & \\
\hline No & 219 & $78.5 \%$ \\
\hline
\end{tabular}

Household member with underlying condition/comorbidities or disability

\begin{tabular}{|lll|}
\hline Yes & 73 & $26.2 \%$ \\
\hline No & 206 & $73.8 \%$
\end{tabular}

Living with elderly people (>65y)

\begin{tabular}{|lll|}
\hline Yes & 96 & $34.4 \%$ \\
\hline No & 183 & $65.6 \%$ \\
\hline Place of work $^{\text {a,b }}$ & & \\
\hline Private clinic & 242 & $86.7 \%$ \\
\hline Private hospital & 218 & $78.1 \%$ \\
\hline Public hospital & 96 & $34.4 \%$ \\
\hline Other & 9 & $3.2 \%$ \\
\hline
\end{tabular}

Frontline worker during COVID-19 pandemic 


\begin{tabular}{|c|c|c|}
\hline Yes & 106 & $38.0 \%$ \\
\hline No & 173 & $62.0 \%$ \\
\hline \multicolumn{3}{|c|}{ Clinical experience in OBGYN (years) } \\
\hline $0-4$ years & 29 & $10.4 \%$ \\
\hline $5-10$ years & 54 & $19.4 \%$ \\
\hline$>10$ years & 196 & $70.3 \%$ \\
\hline \multicolumn{3}{|c|}{ Ever working in an epidemic before } \\
\hline Yes & 60 & $21.5 \%$ \\
\hline No & 219 & $78.5 \%$ \\
\hline \multicolumn{3}{|c|}{ Completing training on COVID-19 } \\
\hline Yes & 111 & $39.8 \%$ \\
\hline No & 168 & $60.2 \%$ \\
\hline \multicolumn{3}{|c|}{ Treating COVID-19 patients } \\
\hline Yes & 136 & $48.7 \%$ \\
\hline No & 143 & $51.3 \%$ \\
\hline \multicolumn{3}{|c|}{ Reluctance to take care of COVID-19 pregnant woman } \\
\hline Yes & 79 & $28.3 \%$ \\
\hline No & 200 & $71.7 \%$ \\
\hline Total (N) & 279 & $100 \%$ \\
\hline
\end{tabular}

- ${ }^{a}$ For questions using checkboxes, the total number of answer selected for a question can be greater than the number of respondents that answered the question (respondents can select multiple answers from a defined list of choices).

- ${ }^{b}$ Questions with multiple choice answers. 


\begin{tabular}{|lllll|}
\hline Table 2: Knowledge domains (N=279) & & & \\
\hline$\#$ & & Poor & Moderate & Good \\
\cline { 3 - 4 } & $\mathbf{n}(\%)$ & $\mathbf{n}(\%)$ & $\mathbf{n ( \% )}$ \\
\hline D1 & $\begin{array}{l}\text { Domain 1: General knowledge about COVID-19 (nb of } \\
\text { items=4) }\end{array}$ & $1(0.4 \%)$ & $22(7.9 \%)$ & $256(91.8 \%)$ \\
\hline D2 & $\begin{array}{l}\text { Domain 2: Specific knowledge regarding pregnancy } \\
\text { and COVID-19 (nb of items=6) }\end{array}$ & $24(8.6 \%)$ & $40(14.3 \%)$ & $215(77.1 \%)$ \\
\hline D3 & $\begin{array}{l}\text { Domain 3: Medicinal treatment and COVID-19 (nb of } \\
\text { items= 5) }\end{array}$ & $24(8.6 \%)$ & $53(19 \%)$ & $202(72.4 \%)$ \\
\hline D4 & $\begin{array}{l}\text { Domain 4: Specific knowledge regarding delivery and } \\
\text { COVID-19 (nb of items=4) }\end{array}$ & $0(0 \%)$ & $14(5 \%)$ & $265(95 \%)$ \\
\hline D5 & $\begin{array}{l}\text { Domain 5: Specific Knowledge regarding } \\
\text { breastfeeding and COVID-19 (nb of items=2) }\end{array}$ & $8(2.9 \%)$ & $51(18.3 \%)$ & $220(78.9 \%)$ \\
\hline & \begin{tabular}{l} 
Overall OBGYN Knowledge score \\
\hline
\end{tabular} & $3(1.1 \%)$ & $93(33.3 \%)$ & $183(65.6 \%)$ \\
\hline
\end{tabular}


Table 3: Knowledge items ( $\mathrm{N}=279)$

\begin{tabular}{ll} 
Correct & Incorrect \\
\hline $\mathrm{n}(\%)$ & $\mathrm{n}(\%)$ \\
\hline
\end{tabular}

\section{Domain 1: General knowledge about COVID-19 ( $\mathrm{nb}$ of items=4)}

K1 The incubation period of COVID-19 -19 is 2-14 days

$274(98.2 \%) \quad 5(1.8 \%)$

K2 COVID-19-19 can persist on surfaces for few hours up to several days.

$235(84.2 \%) \quad 44(15.8 \%)$

There is c

K3 There is currently no vaccine available to prevent infection with COVID-19

$208(74.6 \%) \quad 71(25.4 \%)$

K4 COVID-19 is transmitted through direct contact with respiratory $277(99.3 \%) \quad 2(0.7 \%)$ tract secretions

\section{Domain 2: Specific knowledge regarding pregnancy and COVID-19 (nb of items=6)}

K5 The overall risk of COVID-19 infection in pregnant patient is 115(41.2\%) 164(58.8\%) low.

K6 Pregnant patient COVID-19 positive develop respiratory complications requiring intensive care more than COVID-19 positive non pregnant patient

K7 Risk factors for severe COVID -19 during pregnancy include older maternal age, a high BMI and pre-existing diabetes or hypertension.

K8 COVID-19 positive Pregnant patients are more likely to have preterm labor

$168(60.2 \%) \quad 111(39.8 \%)$

K9 Vertical transmission appears to be uncommon.

$275(98.6 \%) \quad 4(1.4 \%)$

K9 Vertical transmission appears to be uncommon.

K10 Ultrasonography should only be used when it provides medical benefit and when necessary.

\begin{tabular}{ll}
\hline $115(41.2 \%)$ & $164(58.8 \%)$ \\
\hline $168(60.2 \%)$ & $111(39.8 \%)$
\end{tabular}

\section{Domain 3: Medicinal treatment and COVID-19 (nb of items= 5)}

K11 The treatment of COVID-19-19 positive pregnant patients aim to 266(95.3\%) 13(4.7\%) relieve symptoms.

K12 The use of non-steroidal anti-inflammatory drugs (NSAID), as $\quad 197(70.6 \%) \quad 82(29.4 \%)$ ibuprofen, could worsen COVID-19 status.

K13 Low dose aspirin should continue to be prescribed for pregnant $\quad 267(95.7 \%) \quad 12(4.3 \%)$ women as medically indicated.

K14 Maternal immunization continues to be an essential component $\quad 231(82.8 \%) \quad 48(17.2 \%)$ of prenatal care during COVID-19 pandemics

K15 High dose of systemic corticosteroids should be avoided in confirmed or suspected COVID-19 infection. 
K16 Cesarean section is indicated for delivery of all pregnant COVID-19 patients

K17 Timing of delivery in COVID-19 positive pregnant patient is only $\quad 244(87.5 \%) \quad 35(12.5 \%)$ dictated by her obstetrical reasons.

K18 In severe cases of COVID-19 pregnant woman, requiring ICU admission or ventilation, a multidisciplinary team approach $275(98.6 \%) \quad 4(1.4 \%)$ dictate the type and timing of delivery.

K19 If the pregnant woman is scheduled for delivery, she and her support person might be screened for COVID-19 24-48h before $271(97.1 \%) \quad 8(2.9 \%)$ admission.

\section{Domain 5: Specific Knowledge regarding breastfeeding and COVID-19 (nb of items=2)}

K20 COVID-19 can be transmitted through breast milk $230(82.4 \%) \quad 49(17.6 \%)$

K21 COVID-19 19 infected mother can transmit the virus to the baby through respiratory droplets during breastfeeding.

- ${ }^{\mathrm{c}}$ Correct answers (based on the literature available to date).

\section{Table 4: Answers of OBGYN regarding fears items}

\begin{tabular}{|lllll|}
\hline & & Afraid & Neutral & Not afraid \\
\hline F1 & $\begin{array}{l}\text { I am afraid to contract COVID-19 during } \\
\text { occupational exposure. }\end{array}$ & $\mathbf{n}(\%)$ & $\mathbf{n}(\%)$ & $\mathbf{n ( \% )}$ \\
\hline F2 & I am afraid to be very sick if i contract COVID-19. & $179(64.2 \%)$ & $48(17.2 \%)$ & $52(18.6 \%)$ \\
\hline F3 & $\begin{array}{l}\text { I am afraid of transmitting infection to family } \\
\text { member in case i am infected. }\end{array}$ & $265(95 \%)$ & $9(3.2 \%)$ & $5(1.8 \%)$ \\
\hline F4 & $\begin{array}{l}\text { I am afraid of working in facilities where COVID-19) } \\
\text { positive patients are admitted and followed. }\end{array}$ & $118(42.3 \%)$ & $58(20.8 \%)$ & $103(36.9 \%)$ \\
\hline
\end{tabular}

Table 5: Answers of OBGYN regarding impact of COVID-19 on their well being

$\begin{array}{lll}\text { Disagree } & \text { Neutral } & \text { Agree } \\ \mathrm{n}(\%) & \mathrm{n}(\%) & \mathrm{n}(\%)\end{array}$

Impact of COVID-19 on OBGYN wellbeing

11 I think my anxiety (worries) is affecting the quality of $170(60.9 \%) \quad 33(11.8 \%) \quad 76(27.2 \%)$ care anticipated to patient.

12 I feel overwhelmed and burned out by the workload $\quad 82(29.4 \%) \quad 79(28.3 \%) \quad 118(42.3 \%)$ during COVID-19 pandemic. 
Page 24/27 
Table 6: Practices items

Never Occasionally All the time

$n(\%) \quad n(\%) \quad n(\%)$

\section{Practices at personal level}

I clean regularly my hands using soap and water or alcohol based hand rub

I clean regularly my hands using soap and water or alcohol based hand rub before and after treating patient

I change gloves after each patient

$5(1.8 \%)$

$77(27.6 \%)$

197(70.6\%)

I wear the personal protective equipment such as mask, $\quad 2(0.7 \%)$

$2(0.7 \%)$

$9(3.2 \%)$

$268(96.1 \%)$

gloves, face shield, gown....

I routinely clean and disinfect regularly surfaces and equipment's (especially high touched surfaces)

$3(1.1 \%)$

$37(13.3 \%)$

$240(86.0 \%)$

\section{Practices at clinic/facility level}

I request that all staff and visitors wear mask before coming to my clinic

I limit duration of the consultation to the just necessary (according to the medical situation)

$29(10.4 \%) \quad 88(31.5 \%) \quad 162(58.1 \%)$

I limit the number of visitors and/or I employ an extended hours strategy to maintain physical

$12(4.3 \%) \quad 53(19 \%)$

$214(76.7 \%)$

distancing in the clinic and waiting areas

I made some modifications in the waiting room and

clinic space to accommodate physical distancing

I ensure availability of hand hygiene stations for pregnant women before entering the facility or the $0(0 \%)$

$5(1.8 \%)$

$274(98.2 \%)$ waiting area

I maximize use of telehealth modalities (calls, video calls...)

I preserve and prioritize in-person visits for patients with greatest medical needs for in-person examination $25(9 \%)$ $32(11.5 \%)$ $222(79.6 \%)$ (I postpone non-emergent gynecologic/well women appointments)

I screen patients for in-person visits during COVID-19 (pre-appointment phone screening for exposure, COVID$4(1.4 \%)$

$28(10 \%)$ $247(88.5 \%)$

19 symptoms and temperature checking ....)

I request that all health staff at my clinic wear protective clothing

$11(3.9 \%) \quad 169(60.6 \%) \quad 99(35.5 \%)$

$19(6.8 \%)$

$99(35.5 \%)$

161(57.7\%)

\section{Practices with pregnant woman (Counseling \&}


I counsel pregnant woman about the potential risk of

COVID-19 and I document our discussion regarding

$5(1.8 \%)$

$85(30.5 \%)$

$189(67.7 \%)$

prenatal care plan in her medical record

I emphasize measures to prevent COVID-19 for pregnant woman and their families (wearing mask, $2(0.7 \%) \quad 30(12.5 \%)$ $247(88.5 \%)$

hand hygiene, physical distancing, cough etiquette....)

I encourage woman to maintain prenatal care appointments and to took needed vaccines during $8(2.9 \%)$ $35(11.8 \%)$ $236(84.5 \%)$ pregnancy

I advise woman about contraceptive methods during COVID-19

$47(16.8 \%) \quad 119(42.7 \%) \quad 113(40.5 \%)$

I distribute brochures, guidelines regarding COVID-19 and pregnancy

I encourage my patients to notify me and the health authorities of any potential exposure to COVID-19

$125(44.8 \%) \quad 100(35.8 \%) \quad 54(19.4 \%)$

$2(0.7 \%)$

$22(7.9 \%)$

$255(91.4 \%)$

\section{Figures}

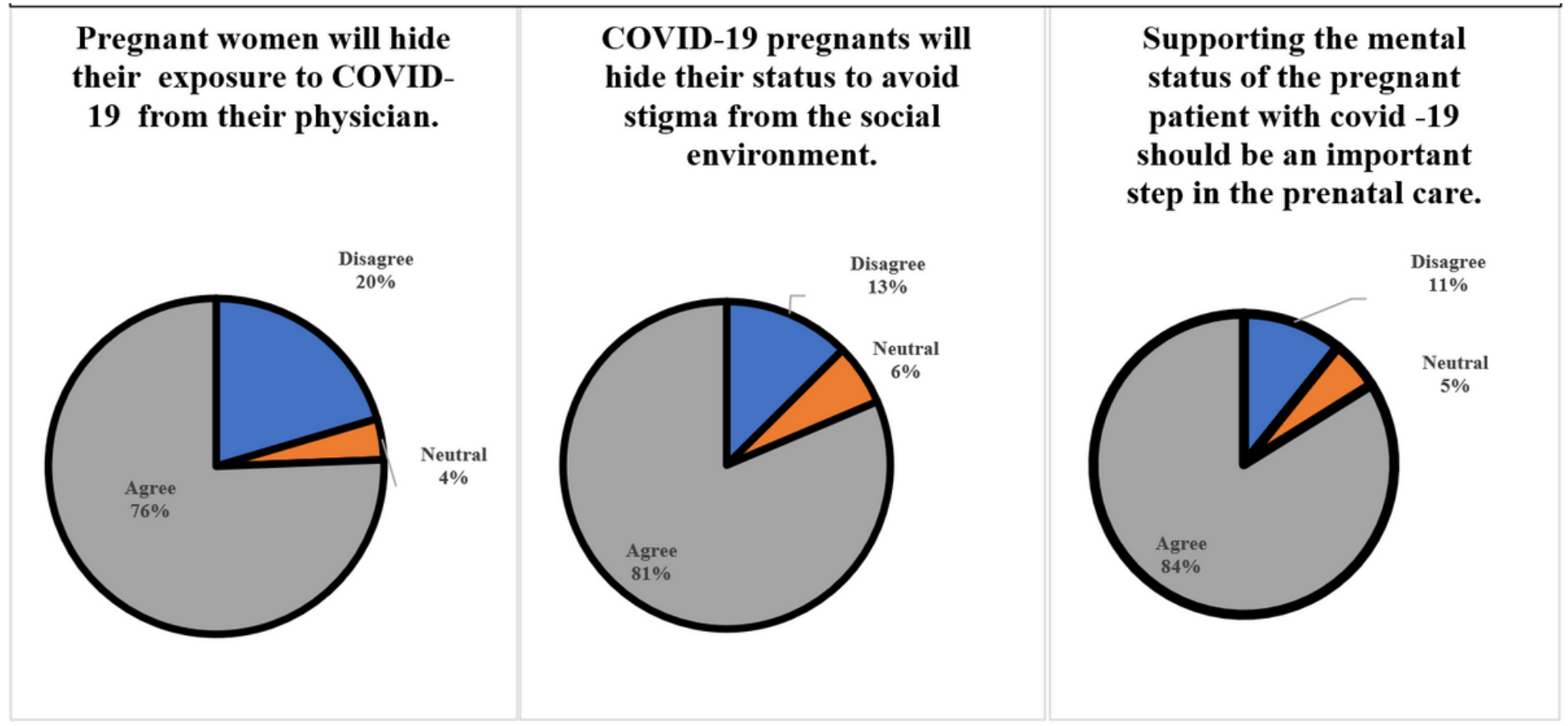

Figure 1

Attitudes of OBGYN's towards pregnant woman 


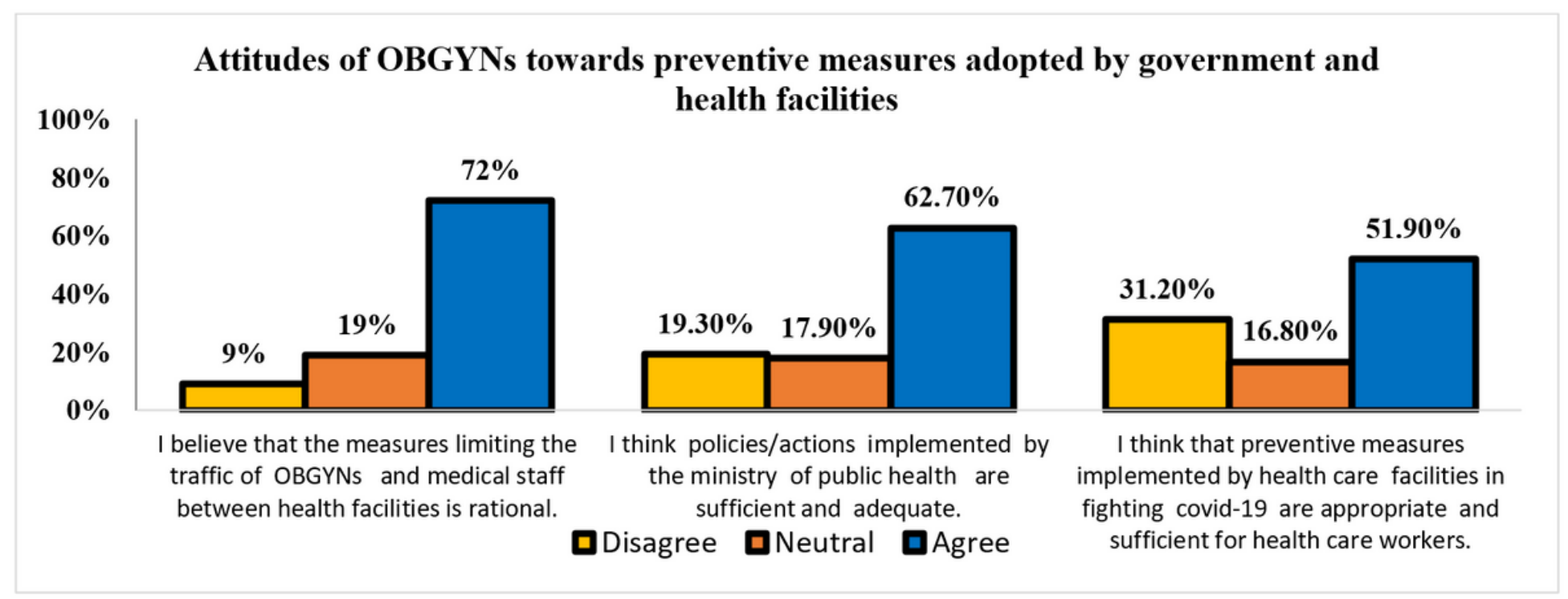

Figure 2

Attitudes of OBGYN's towards preventive measures adopted by government and health facilities

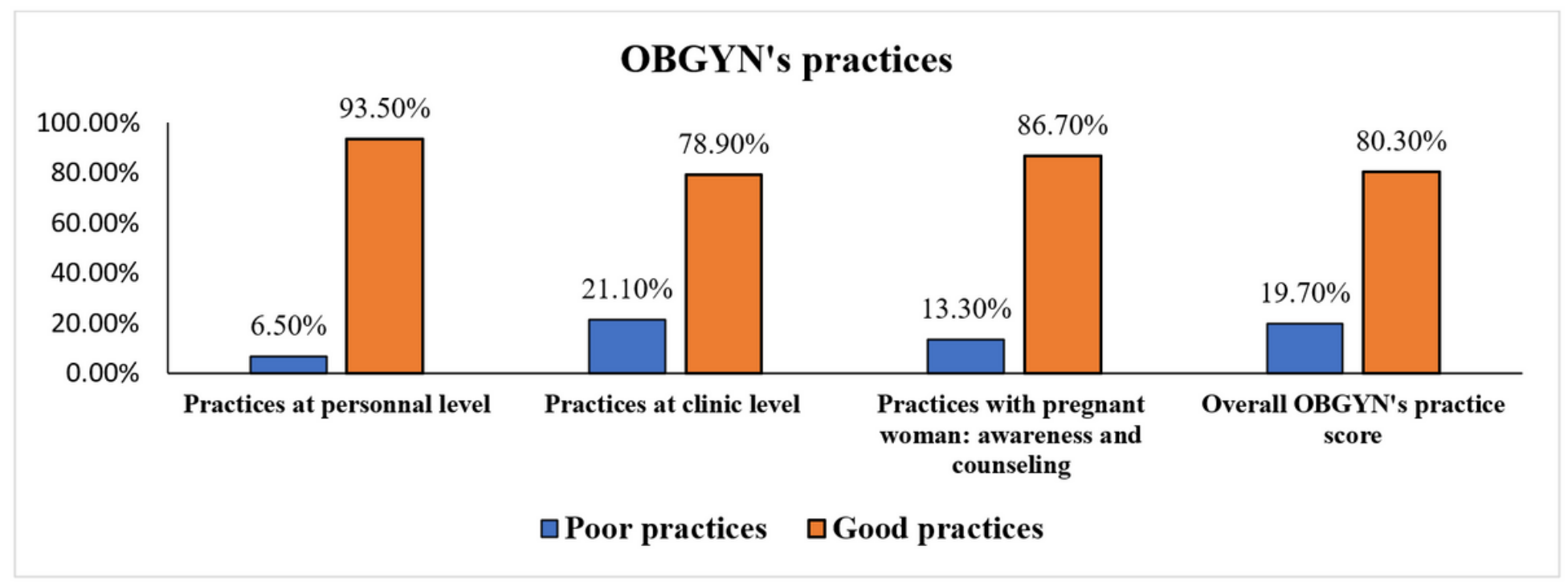

Figure 3

OBGYN's practices

\section{Supplementary Files}

This is a list of supplementary files associated with this preprint. Click to download.

- OBGYNKAPSupplementfile.docx 